\title{
Review of Anthropomorphic Head Stabilisation and Verticality Estimation in Robots
}

\author{
Ildar Farkhatdinov ${ }^{1,2}$ Hannah Michalska ${ }^{3}$ Alain Berthoz ${ }^{4}$ Vincent Hayward ${ }^{5}$ \\ 1 School of Electronic Engineering and Computer Science, \\ Queen Mary University of London, London, United Kingdom, \\ i.farkhatdinov@qmul.ac.uk \\ 2 Department of Bioengineering, Imperial College London, London, United Kingdom \\ 3 McGill University, Montréal, Qc, Canada \\ 4 Collège de France, Paris, France \\ 5 Sorbonne Universités, UPMC Univ Paris 06, Institut des Systèmes Intelligents et \\ de Robotique, Paris, France
}

\begin{abstract}
In many walking, running, flying, and swimming animals, including mammals, reptiles, and birds, the vestibular system plays a central role for verticality estimation and is often associated with a head stabilisation (in rotation) behaviour. Head stabilisation, in turn, subserves gaze stabilisation, postural control, visual-vestibular information fusion and spatial awareness via the active establishment of a quasi-inertial frame of reference. Head stabilisation helps animals to cope with the computational consequences of angular movements that complicate the reliable estimation of the vertical direction. We suggest that this strategy could also benefit free-moving robotic systems, such as locomoting humanoid robots, which are typically equipped with inertial measurements units. Free-moving robotic systems could gain the full benefits of inertial measurements if the measurement units are placed on independently orientable platforms, such as a human-like heads. We illustrate these benefits by analysing recent humanoid robots design and control approaches.
\end{abstract}

\section{Introduction}

Spatial awareness is crucial for free-moving robots. For instance, legged humanoid robots must be aware of their body motion and orientation with respect to gravity in order to maintain stable posture and gait. Recent technological developments in sensing and control for robotic systems contributed to the appearance of new humanoid robots with human-like (anthropomorphic) behaviours. However, the performance of robots is still lagging far behind the abilities of biological systems. For example, it is clear that that a powerful and adaptive sensorimotor control system enables humans, for example, to balance on fallen trunks or run on sandy beaches, not mentioning the locomotion prowesses of felines, of mountain goats, and so on.

The purpose of this chapter is to examine the phenomenon of head stabilisation displayed by biological and robotic systems and the importance of such 
behavior in motion sensing and body balancing. Head stabilisation has been extensively studied by neuroscientists and recently several robotic systems have adopted this human-like control to improve inertial sensing, gaze and postural control, and interaction qualities of the robots. Functions of the vestibular system and its primary roles are first reviewed in Section 2. Head stabilisation in humans is next discussed in Section 3, which is then followed by the overview of inertial sensing and head stabilisation in robotics systems in Sections 5 and 6 .

\section{Sensing body movements in animals and robots}

\subsection{Sense of motion}

Humans and animals have a complex multisensory system which provides the central neural system (CNS) with information about the external world. For us, like for other living creatures, it is important to understand the spatial orientation and localization of our bodies with respect to the objects of the external world. This can be accomplished by the help of diverse sensory systems: vision, audition, tactile, proprioception and vestibular. Change of visual flow in the retina would indicate that the position of the body changed with respect to an observed environment. Change of difference in acoustic flow in the ear would indicate that the body moves with respect to a sound source. Likewise, tactile and proprioceptive inputs would signal position change of the body if the latter interacts with an environment. However, independent sensory information of one modality only may not be sufficient for correct motion perception. For instance, change of visual flow can be caused by movements of eyes, sound source movement may be estimated wrongly if the head rotates, etc. Then, the problem is solved by multisensory integration [45]. The CNS can efficiently process multisensory information and provide the brain with a proper motion sensation. Visual, audition, tactile and proprioception sensory inputs, excluding vestibular, can be easily put out of action while vestibular inputs will always be there. Even in case of zero gravity vestibular organs will respond to linear accelerations and angular velocities of the body motion. It is thus reasonable to claim that the vestibular system plays a key role in perceiving self-motion. The principles which govern vestibular organs and their roles for postural control will be described next.

\section{$2.2 \quad$ Vestibular system}

The vestibular system detects motion of the head in space and generates reflexes that are crucial for survival, such as stabilising gaze and maintaining head and body posture. In addition, the vestibular system provides its owner with a subjective sense of movement and orientation in space. The vestibular sensory organs are located in close proximity to the cochlea, as shown in Figure 1A. The vestibular system consists of two types of organs: the two otolith organs and the three semicircular canals. Two types of otolith organs (the saccule and utricle) 
sense linear acceleration which includes gravitational and translational forces. Semicircular canals sense angular velocities in three planes. Receptor signals are sent through the vestibular nerve fibres to the neural structures that are responsible for eye movements, posture and balance control. As a result, the vestibular organs constitute our sixth sense - the sense of motion which allows us to perceive and control bodily movements [16]. Vestibular processing is highly multimodal, for instance, visual/vestibular and proprioceptive/vestibular sensory inputs are dominant for gaze and postural control, but at the same time vestibular system itself plays an important role in everyday activities and contributes to a various range of functions $[5,35]$.
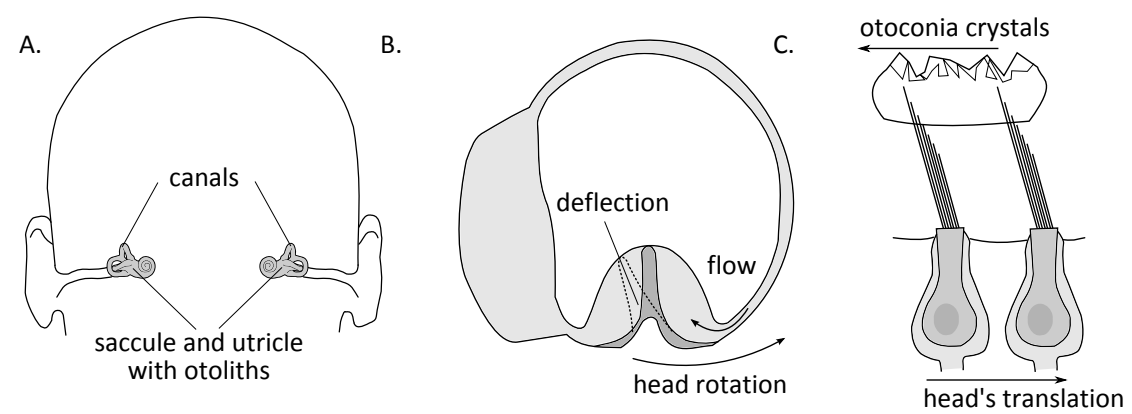

Fig. 1. The vestibular organs located in each inner ear (A). Main organs are three almost orthogonal semicircular canals (superior, posterior and lateral) and two otolith organs (utricle and saccule). Semicircular canals measure angular velocity of the head (B). Otolith organs measure linear gravitoinertial acceleration (C).

Each inner ear has three semicircular canals arrayed approximately at right angles to each other. The real geometry has evolved during evolution depending upon the need of each species [37]. Semicircular canals are sensitive to angular accelerations [121]. Each canal is comprised of a circular path of fluid continuity, interrupted at the ampulla by a watertight, elastic membrane called the cupula [5]. Fig. 1B shows a schematic view of one semicircular canal. Each canal is filled with a fluid called endolymph. When the head rotates in the plane of a semicircular canal, inertial forces causes the endolymph in the canal to lag behind the motion of the head [28]. The motion of the endolymph applies pressure to the membrane of the cupula and its deflection causes shearing stress in the hair cells [28]. The corresponding electrical signals are generated and transmitted through neurons in a way similar to the way it is done in the otoliths. Although the semicircular canals respond to angular acceleration, the neural output from the sensory cells represents the velocity of rotation. This suggests that the input signal is integrated due to the mechanics of the canals, mainly because of the increase in viscous properties of the fluid in a tight canal $[36,76]$. The vestibular system transmits the components of the angular velocity of the head to the CNS which forms a three-dimensional angular velocity vector describing 
the body motion. More advanced experimental studies with human subjects supported the torsion-pendulum representation of the canal dynamics $[75,68,107]$. In [139], it was suggested to model semicircular canals with the heavily-damped second order system which behaved as an angular-velocity meter.

The otolith organ comprised utricle and saccule, both sensitive to linear acceleration of the head in linear motion and static tilt in the vertical planes. The utricle and the saccule are arranged to respond to the motion in three dimensions. When the head is upright, the saccule is vertical and it measures linear accelerations in the sagittal plane, specifically movements up and down. The utricle is horizontally oriented and measures accelerations in interaural transverse (horizontal) plane (anterio-posterior and medio-lateral accelerations) [70]. Both, the utricle and the saccule, contain sheets of hair cells a sensory epithelium (macula). An otolithic membrane (otoconia) composed of calcium carbonate crystals sits atop of hair cells. Fig. 1C shows the simplified view of otolithic hair cells during an accelerated motion to the right. In response to linear acceleration, the crystals are left behind due to their inertia. Linear acceleration of the head causes otoconia's motion which in turn causes shear forces acting on the hair cells. In addition during head tilt they can measure the changes of head angle with respect to the gravity vector. Complex molecular level mechanic-electrochemical mechanism of interaction between hair cells results in the generation of electrical signals which are sent to the neural structures for further processing [28]. The displacement of the large saccular otolith of a ruff was measured in [39] and the dynamic identification suggested that the mechanics of the otolith could be described by a critically damped second-order system with a resonant frequency of $50 \mathrm{~Hz}$. A recent model of the geometry of the utriculus and sacculus has suggested that the curved shape of the striola (which contains the dynamic sensory cells) are in fact the projection of a curved virtual striola giving the ensemble of the two sensors the capacity to measure directly the 3D acceleration vector of the movements for the head in space [41].

\subsection{Sensory data integration and processing}

Sensory outputs from otoliths and semicircular canals are processed in neural systems on different levels. Some information is processed in low-level neural networks, while some are directed to CNS where integration with other sensory modalities may occur.

L. R. Young with his group proposed an optimal estimator model in [21]. A concept of the internal model was introduced which comprised the dynamic model information about the sensory organs and head-neck system. This internal model was considered to be known for the CNS. L. R. Young used an optimal estimator (Kalman filter) to estimate the human's orientation based on the outputs from visual, vestibular, proprioceptive and tactile sensory systems. Assumptions about sensor dynamics and noise statistic from internal model were used to correct the estimated states which represented spatial orientation. Estimated states, called as perceptions, contained angular orientation of the head, its angular velocity, inertial translation and inertial velocity. 
In similarity with [21], an internal model based approach was proposed by D.M. Merfeld and colleagues in [99]. Their sensory integration model was based on simulating the neural processing of gravito-inertial cues. In $[151,101]$ experiments were performed to analyse the vestibular-ocular response during tilt and rotation of the head and the body. The presented experimental findings were consistent with the hypothesis that the nervous system resolves the ambiguous measurements of gravitoinertial forces into neural estimates of gravity and linear acceleration. A human model for this vestibular signals processing was presented in [100]. A linear system was used to model the semicircular canals' dynamics, and a simple gain (identity matrix) was used to model the otoliths. It was suggested that the CNS is able to perform the operation of gravitational vector transformation (rotation) from the world frame to the head's frame.

More recently, J. Laurens and J. Droulez built a Bayesian processing model of self-motion perception in [90]. It was proposed that the brain processes these signals in a statically optimal fashion, reproducing the rules of Bayesian inference. It was also proposed that the Bayesian based processing uses the statistics of natural head movements. The outputs of semicircular canals and head's angular velocity were assumed to be subjects to Gaussian noise. By using particle filtering, a three dimensional model of vestibular signal processing model was developed based on optimal estimation. The model was successfully tested by computational experiments, as well, it was proved to be efficient in modelling the vestibulo-ocular reflex.

Among the vestibular information processing models mentioned above, the concepts of internal model and estimator or observer are crucial for the system description. Detailed reviews of existing vestibular information processing models can be found in $[96,126]$. Interestingly, in robotic systems, the concept of the internal model has been known for decades, since the early works of E. Kalman in linear filtering [82] and D. Luenberger in state estimation for linear systems [95].

\subsection{Roles of the vestibular system}

Humans rely on the multiplicity of sensory inputs and sophisticated anticipatory mechanisms to solve the control problems subserving standing, walking, running, jumping, dancing, and so on. Vestibular inputs play a central role in all these tasks, which are achieved through a combination of postural movements and forces and torques exerted against the environment. Better understanding of the functioning of the vestibular system may have important implications in design and control of robotic systems.

Vision. In humans, the head-located vestibular system is known to participate in a number of functions that include gaze stabilisation through the vestibulo-ocular reflex $[18,17,57,40]$. The vestibulo-ocular reflex stabilises the gaze to ensure clear and still vision. It is a reflex of eye movement that stabilises the image projected on the retina during head movements. Produced eye movements are in the direction opposite to head movements. The reflex has both rotational and translational components which are driven by semicircular canals and otoliths, respectively. 
Self-motion perception. The vestibular system is a key sensory organ that enables perception of body motion [19]. A human with a healthy vestibular system can always tell if the body is moving even if other senses such as vision, audition are absent. Vestibular system provides us with the ability to distinguish between self-generated motion and the external one. It has been shown that vestibular only information is sufficient to reconstruct current and past passive body locations $[77,78]$.

Balance. The vestibular system plays a dominant role in the coordination of postural reflexes, such as vestibulocollic reflex. The vestibulocollic reflex is responsible for maintaining head and body posture. This reflex stabilises the head with respect to inertial space. It produces commands to move the head in the direction opposite to the direction of the actual velocity of the head [47, 10]. Another important role of the vestibular system is vestibulospinal reflex which coordinates head and neck movement with respect to the trunk of the body. The goal of the reflex is maintaining the head in an upright position [3]. Together, vestibulocollic and vestibulospinal reflexes are responsible for selfbalancing control $[145,146,127,2,98]$.

Perception of verticality. The vestibular system is the principal sensory system which is able to perceive perceive gravitational forces. When the body is motionless, otoliths measure the gravitational acceleration vector, whose components render the sense of absolute verticality [20, 149]. Knowledge of gravitational verticality is essential for balancing and posture control, as it allows to determine spatial orientation of the body [30].

Frame of reference. The vestibular system, like embodied inertial sensors, provides the CNS with a head-centred frame of reference. It may be suggested that low-level balance and posture control is realised in this frame of reference. Spatial transformations from head-fixed and world inertial frame can be performed based on vestibular system measurements. Therefore, this embodied frame of reference is directly related to the world inertial frame and enables the neural system to perform stable posture control independently from other sensory inputs, such as tactile or proprioception information on ground inclination. In this way, ground-independent posture and balance control can be implemented.

\section{Head stabilisation behaviour in animals and humans}

Various human motion experimental studies showed that humans stabilise their heads while performing locomoting, balancing or other postural tasks. It has been initially proposed by T. Pozzo and A. Berthoz that humans stabilise their heads in rotation for different locomotor tasks, such as free walking, walking in place, running in place and hopping [115]. In the experiments with ten healthy subjects humans stabilised their heads in such a way as the maximum angular amplitude of Frankfort's plane (plane of horizontal semicircular canals) did not exceed $20^{\circ}$. It was suggested that such stabilisation is probably due to a cooperation between both the measurement of head rotations by the semi-circular canals and 
the measure of translations by the utriculus and sacculus (otolith organs). The plane of the stabilisation is determined by the task; it can vary and be controlled by gaze. Further experiments showed that total darkness does not significantly influence the stabilisation of the head, which stresses the importance of this behaviour in the coordination of the multiple degrees of freedom of the body during gait [116].

Later in [117], it was shown that head stabilisation occurred also in the frontal plane during the maintenance of monopodal and bipodal equilibrium on unstable rocking platforms. The head remained stable relative to the vertical, despite large translations in the frontal plane. Head angular stabilisation close to vertical orientation was essential for effective postural control during those complex balancing tasks.

Some recent behavioural studies by A. Berthoz and colleagues showed that head orientation is anticipated during locomotion relatively to walking direction $[64,113,73]$. This may suggest that head orientation and gaze stabilisation is important for motion planning during locomotion, and both visual and vestibular cues can be processed by the CNS in a better way if the head is stabilised and oriented towards the walking direction. Additional studies suggested that the motion of the head, together with gaze control, is closely related to optimal postural control during locomotion [6].

In [84], a control mechanism model for head stabilisation was described. Angular velocities of the head and trunk in space were recorded in seated subjects during external perturbations of the trunk. The passive mechanics of the head was changed by adding additional mass in different experimental trials. It was shown that head stabilisation in a horizontal plane in yaw orientation did not differ much with respect to the changed inertia of the head. For pitch motion, the response of the head-neck stabilisation controller was changed when additional mass was added to the head. However, in all the cases subjects were stabilising their heads while their trunk was perturbed.

In [25], balancing on a moving platform with subjects with and without the bilateral vestibular loss was studied. Results showed that subjects with vestibular loss were unable to perform that task properly, and their trunk and head were not stabilised in space, while healthy subjects were stabilising their heads regardless to the motion of the platform.

Similar to humans, head stabilisation was observed in many other animals. For instance, head stabilisation was reported for cats [61]. Similar head stabilisation was found in monkeys during locomotion [148]. The head was stabilised and absolute values of pitch and roll head movements did not exceed $7^{\circ}$. The similar result was obtained for running monkeys [42]. For horses, a weaker head stabilisation effect was reported [43].

Head stabilisation was observed for birds, as well. In [65], head stabilisation in chickens during jumping and walking on surfaces of different slopes. Results suggested that the head was stabilised during locomotion but the angle of stabilisation increased with increasing downwards slope of the walking surface and decreased with increasing upwards slope. Head stabilisation was reported for 
other birds such as herons, as well [83]. In most of the cases, different types of herons stabilised their heads' spatial orientation and location while their body was disturbed by harmonic oscillations. Head bobbing during locomotion is a very well-known behaviour for birds like pigeons, egrets, whooping cranes [55, $38,34,56,136]$. Head-bobbing behaviour results in stabilisation of the head to the surroundings for specific phases of the locomotion cycle. This bobbing is coupled with the locomotion and is mainly under the control of visual system inputs $[55,106]$.

In the studies mentioned above, it was shown that orientation of the head is naturally stabilised during locomotion, balancing. These studies emphasized the importance of head stabilisation as a part of the general postural control in humans and animals. In some cases, head stabilisation may be related to the task performed by a human, but naturally, the head stabilisation may be the result of vestibular-ocular interactions, as well [69]. However, in all of these cases vestibular information is important for the head-neck control system, and sometimes it is the only source of information available [23]. One of the key roles of head stabilisation is related to the establishment of a stable reference frame in which spatial perception, multiple sensory integration, and postural control are performed. The stable frame of reference based on vestibular information provides the brain with a mobile reference frame, which, in cooperation with vision and gaze allows a 'top-down' control of locomotion $[15,8]$.

\section{Sensing motion in robots with inertial sensors}

\subsection{Inertial sensors}

In robotic systems, inertial sensors play a role similar to the vestibular system in humans and animals. In this subsection, we give a short introduction to inertial sensors and their applications in robotic systems. Modern inertial sensors are electromechanical transducers which measure translation and rotation of their bodies. Recent technological developments provided robotics with various types of inertial sensors $[12,144]$. The most common inertial sensors are accelerometers, gyrometers and inclinometers. Very often they are combined together and then they are called inertial measurement units (IMU).

An accelerometer measures linear acceleration which is a result of linear forces and gravitational forces applied. Modern accelerometers are usually implemented in MEMS based electronic circuits and can have sensitivity in one, two or three axes. Some examples of different technologies used in accelerometer design can be found in $[87,4,108,26,118]$. In a typical accelerometer, a rigid body is constrained to linear translation inside the frame of the sensor. The body is attached to the frame with a spring of a known stiffness. Assuming linear stiffness, low friction and small displacements acceleration of the sensor's body can be calculated from the body's displacement. Importantly, accelerometers react to both translational and gravitational accelerations. In absence of translational motion, they can thus be used as tilt meters (inclinometers). However, this causes a significant problem for acceleration sensing in robotics which 
is often called gravito-inertial ambiguity. It is impossible to distinguish between translational and gravitational components of acceleration when a robotic body is being accelerated in translation and rotation at the same time.

An inclinometer is a sensor used to measure the absolute angular orientation of a body with respect to gravitational acceleration. The physical principle of sensing is the same as for the accelerometer, but an inclinometer's primary function is to measure angular orientation with respect to the gravitational acceleration vector. A very simple type of inclinometer can be implemented with a pendulum which tends to keep its verticality, and in steady state, it is aligned with the gravitational acceleration vector. The measurement is an angle between the pendulum and the frame of the sensor. This angle is usually measured by magnetic, optical or electromechanical angular encoders attached to the joint between the sensor frame and the pendulum. Usually, a moving body in an inclinometer is damped to achieve convergence and steady measurements. In the cases when the frame of the sensor is accelerated, the measurement from the inclinometer is no longer tilt-only related, and the sensor behaves in the same way as the tilted accelerometer as it responses both to translational and gravitational acceleration. Modern inclinometers use different electromechanical, magnetic, hydraulic, optical and other physical effects. Some design examples can be found in $[102,97,92,150]$.

A gyrometer (or a gyroscope) is a sensor for measuring the angular velocity. Modern gyrometers, similar to accelerometers are implemented in electronic circuits based on MEMS technology $[66,105]$. A common MEMS based gyrometer measures angular velocity by means of Coriolis acceleration [60].

\subsection{Verticality estimation methods}

Verticality estimation is a crucial task for the stability of mobile robotic systems. In particular humanoid robots and other walking machines require accurate knowledge of verticality to perform balancing and postural tasks in the gravitational field. In this subsection, we look into some basic methods of verticality estimation methods used in robotic systems. Here we limit our review to approaches which use inertial measurements only. Theoretically, simple time integration of gyrometers output will provide us with the angular orientation of a body (robot) in space. However, initial conditions have to be known, which is not the case for many real-life applications. Another problem with rate sensors is bias, which changes with time, temperature and other conditions. An accelerometer can be used as a tilt sensor to measure the vertical orientation of the robot in the cases when the linear acceleration of the robot is zero or negligible. But most of robotic systems such as humanoid robots are moving continuously with variable velocities and are the subjects to unpredictable mechanical impacts. Generally speaking, inertial sensors are noisy because they pick-up vibrations that become added to the low-frequency components of interest of the acceleration and velocity signals. Gyroscopic measurements also suffer from bias and are highly sensitive to dynamic errors. To combat these problems, different ap- 
proaches to the design state observers and sensor fusion methods have been proposed to improve inertial measurements [13].

In [4], a nonlinear regression model to improve the accuracy of a low- $g$ MEMS accelerometer was proposed. It was assumed that accelerometer was not translated and its measurements provided the tilt information only. Similarly, in [119] accelerometer was used as a tilt sensor. Accurate tilt sensing was achieved with a linear kinematic model which included scale factor, bias and misalignment. It was assumed that the body does not perform any translational motion which makes this approach limited to rotations only. Gyrometer and accelerometer measurements were used together with a Kalman filter for sensors alignment and calibration errors compensation in [67]. Similar to previous cases only rotations were considered.

The vertical orientation of a flying robot was estimated in [9]. The measurement system included an inclinometer and a rate gyro. The data coming from the sensors was fused through a complementary filter, which compensated the slow dynamics of the inclinometer. It was assumed that the robot did not perform fast accelerated motions.

A sensor fusion approach for verticality estimation in mobile robots which are translated and rotated simultaneously was proposed in [138]. To resolve the ambiguity of translational and gravitational components in measurements the odometry of the mobile robot was used. Angular measurements from the robot's wheels, joints and knowledge of its kinematics were used to provide the estimator with additional information about the robot's orientation.

In [120] drift-free attitude estimation for accelerated rigid bodies was described. The attitude estimation problem for an accelerated rigid body using gyros and accelerometers was solved with a switching algorithm which employed Kalman filters. Switching was performed based on the values of estimated linear acceleration of the body. The main idea of the algorithm was to consider the translational accelerations as disturbances that were measured partially by the accelerometer. When the acceleration of the body was too high, the accelerometer measurements were considered completely unreliable to provide verticality measurements switching algorithm forced the estimator to rely on the rate gyros by setting the accelerometer noise covariance matrix to infinity.

Additional inclination sensor was used in [91]. A state estimation technique was developed for sensing inclination angles using a low-bandwidth tilt sensor along with an inaccurate rate gyro and a low-cost accelerometer. The model of rate gyro included an inherent bias along with sensor noise. The tilt sensor was modelled as a pendulum and was characterized by its own slow dynamics. These sensor dynamics was combined with the gyrometer model to achieve highbandwidth measurements using an optimal linear state estimator. Acceleration of the system in the inertial frame was measured by additional accelerometer and was considered as a known input for the estimator. However, in many robotic systems knowledge of the acceleration vector expressed world inertial frame is unavailable or requires additional global measurements. 
In [46] acceleration of an unmanned aerial vehicle expressed in the world frame was estimated by an airflow sensor. This estimation together with the raw measurement from accelerometer was used to calculate the gravitational component of the acceleration. But the performance of the air flow sensor and may not be suitable for human-like walking robotic systems.

A multiple accelerometer based sensory system was used for attitude estimation of a rigid body in [135]. A set of accelerometers was attached to various locations of a rigid body and an optimal linear estimation algorithm was developed for determining pitch and roll of the body fixed at a pivot. The estimated tilt angles values were filtered based on additional angular velocity measurements.

As shown above, the attitude estimation problem for robotic systems is a complex one and often requires nonlinear state estimation techniques to solve it [33]. One of the key problems in verticality estimation based on inertial sensors only is the ambiguity in acceleration or inclinometer measurements. Additional techniques are required to separate the gravitational component of acceleration from translational one. In many cases, extra sensors may facilitate the solution. For example, global positioning system (GPS) was used in [59]; magnetic field sensors were utilized in $[103,54]$, additional bearing information was required in [11]; landmark measurements were used in [141], Earth horizon sensor was utilized in [63], active vision system was employed in [22]. In humanoid robots, it was shown that inertial sensing can be efficiently used to estimate the robot's attitude when combined with body joint and links relative position sensors and legs contact information [14,123] or based on additional information from an external range sensor [48]. Like robotic systems, living creatures are solving the same problem of estimation gravitational verticality using a limited set of sensors.

\section{Anthropomorphic head stabilisation in human-like robots}

\subsection{Location of inertial sensors in humanoid robots}

In living creatures, past and present, the vestibular system is located in the head which is explained by their evolutionary development and specific roles of vestibular organs. In robotic systems, a common design wisdom wants that the inertial sensors to be located close to the centre of mass of a humanoid robot. Most of the humanoid robots have their IMU located in the main body close to their centre of mass. Locating an IMU close to the centre of mass provides information about the motion of the centre of mass, of rotations about it, and allows the application of simple models (inverted pendulum) for locomotion and balancing control tasks. In Table 5.1, we summarised our survey results on the location of inertial sensors in some of the humanoid robots used in research.

As we can see from the table, most of the robots have their IMU, or, we may call it, artificial vestibular system, in the main body: between hip joints, torso, 
Table 1. Location of the inertial sensors in the humanoid robots

\begin{tabular}{llll}
\hline \hline Robot's name & \multicolumn{2}{l}{ IMU location } & Reference Year \\
\hline Cog & head & {$[24]$} & 1999 \\
ASIMO & upper body & {$[124,74]$} & 1999 \\
ATLAS (DASL-1) & pelvis & {$[79]$} & 2002 \\
H7 & upper body & {$[81]$} & 2004 \\
KHR-3 HUBO & torso & {$[112]$} & 2005 \\
FHR-1 FIBO & hip & {$[147]$} & 2006 \\
KHR-2 & torso & {$[85]$} & 2006 \\
CB & head \& torso $[31,71]$ & 2007 \\
iCub & head & {$[137,111]$} & 2007,2012 \\
MAHRU-R & pelvis & {$[29]$} & 2008 \\
ARMAR-III & head & {$[7]$} & 2008 \\
Lola & upper body & {$[27,93]$} & 2009 \\
Nao & torso & {$[62]$} & 2009 \\
AAU-BOT1 & trunk & {$[133]$} & 2009 \\
Sarcos Primus & hip & {$[131]$} & 2010 \\
KOBIAN & head & {$[86]$} & 2012 \\
WABIAN & head & {$[49]$} & 2012 \\
ROMEO & head & {$[114]$} & 2014 \\
COMAN & waist & {$[1]$} & 2014 \\
MOMARO & head & {$[125]$} & 2016
\end{tabular}

trunk or pelvis. Only some robots have their IMU located in the head. In robots like Cog, [24], and iCub, [137], the IMU is located in their heads. However, the original design of these robots did not include locomotion functionality, since they were mainly used for human-robot interaction and computational neuroscience studies. The ARMAR-III robot, [7], has its inertial sensors in the head, but the lower body of the robot is based on a wheeled platform, which makes it very different from legged biological systems. To our knowledge, very few physical humanoid robots use inertial sensors in the head for posture control during locomotion and balancing. Another example, the CB humanoid, [31,71], uses two IMUs: one in the head and another one in the torso. Information from both of them is used to coordinate eyes, head and torso movements [132]. In [86] and [49], robotic heads were equipped with IMUs which were used for head stabilisation during locomotion. In [86], direct measurements of the head's angular orientation were fed back to linear control of the head. However, the performance of this head stabilisation system was quite low, because there were no filtering or estimation methods used for sensory data processing. In [49], a feedback learning algorithm was employed to stabilise the head orientation independently from the trunk motion. A neural network was used to learn the unknown head dynamics. More recent robotic systems used IMU located in the head for cooperative headneck movements and gaze control, which enabled the humanoid robot to achieve human-like gaze and head control behaviour. 


\subsection{Head stabilisation in robots}

In robotic systems, inertial sensors provide control systems with important information on actual robot's state such as its body's acceleration, velocity, orientation. This information is important for maintaining stable motion while performing a required task. Accelerometers are often utilized as tilt sensors and gyroscopes are used to measure the change in the robot's orientation. Next, we describe some of the robotic applications in which head stabilisation plays an important role to achieve better performance and human-like behaviour.

Balancing and walking. Classical humanoid robot locomotion strategy is based on zero moment point (ZMP) control originally proposed by M. Vukobratovic [143]. In this case, knowledge about the robot kinematic configuration and mass distribution was sufficient for stable locomotion. Modern humanoid robotics requires robots to move faster and perform more dynamic tasks which require the use of additional sensory information, such as inertial measurements to enhance balance control of the robot.

For example, in [133], development of AAU-BOT1 humanoid robot is described. In this robot, an IMU was placed in the trunk and was used for balance control during locomotion. The orientation of the robot measured by accelerometers and gyroscopes and foot-ground force reactions measured by force sensors were considered to be the dominant information for walking controller.

Tilt and angular velocity of humanoid's torso were used for balance and walking control in [85]. Tilt was measured by accelerometers attached to the torso of the robot. Direct measurements were used by torso roll and pitch controller and predicted motion controller to avoid tilt over situations.

Inertial measurement unit was used for humanoid balance control in [31, 71]. Three-axis accelerometer and gyro were attached close to the centre of mass in the trunk of the robot. Angular velocity information was used for the centre of mass measurement and calculation of the desired ground interaction force direction for maintaining a stable posture.

Balance control of humanoid robot was described in [29]. The robot's body was equipped with an IMU for measuring trunk's angular velocity and orientation in space. This information was used for the robot's actual posture calculation together with the measurements from joint sensors.

Biologically inspired postural and reaching control for the humanoid robot was presented in [134]. A humanoid robot was equipped with accelerometer and gyrometer in the head. Normal and tangential contact forces between the robot and the platform were measured by force sensors. Unlike in most of the humanoid research literature, in [134], the platform's (ground's) non zero inclination was considered to be unknown. Unknown ground tilt and disturbance forces were estimated based on measurements from accelerometer and gyrometer. Estimation was implemented by Kalman filtering. It was claimed that inertial sensors and their signals processing could be called as the artificial vestibular system of the humanoid robot [134]. In [114] a sophisticated version of IMU and eye control integration was presented for a humanoid robot. The system was based 
on coordinated human-like control of head-neck and gaze system which enabled to achieve natural saccadic movements in the head's frame of reference.

Gaze and head stabilisation. In many recently developed human-like robots, the vestibulo-ocular reflex is realised for gaze stabilisation. Common human-like robot's head is equipped with two video cameras. Stereo information from these cameras is used for robot's localization and visual perception of the environment. For instance, it may be very important for planning grasping tasks. This visual flow should be stabilised by implementation gaze stabilisation like it is done in biological systems. In addition, this gaze stabilisation behaviour makes a humanoid robot's more natural and realistic which is important in human-robot interaction scenarios.

One of the first humanoid ocular motor control systems was described in [128, 129]. A biomimetic gaze stabilisation based measurements from three gyros in the head of the robot was implemented. Feedback-error learning algorithm with neural networks was implied to achieve human-like vestibulo-ocular and optokinetic reflexes. Similarly, in [110], the humanoid robot's head was equipped with an IMU and video cameras. Measurements from the IMU and analysis of visual flow from cameras were used for achieving efficient visual stabilisation. Inertial sensors provided short latency measurements of rotations and translations of the robot's head. Visual flow information provided a delayed estimate of the motion across the image plane. A self-tuning neural network was used to learn to integrate visual and inertial information and to generate proper ocular-motor control signals.

Vision only based gaze stabilisation was presented in [58]. Adaptive frequency oscillators were used to learn the frequency, phase and amplitude of the optical flow from the robot's cameras during locomotion. The developed vision-based control was used for gaze stabilisation during periodic locomotion and for visual object tracking.

In [31], a three-axis gyrometer and an accelerometer in the head of the humanoid were used for controlling the gaze and implementing visual attention system. In [94], biomimetic eye-neck coordination control was proposed and used for visual target tracking. Proprioceptive feedback from neck joints and vestibular signals from inertial sensors in the head were processed together. The control system was tested with the iCub humanoid robot.

\section{Benefits of head stabilisation in robots}

\subsection{Towards a robotic vestibular system}

In the previous section, we have presented some example of robots with IMUs located in their heads. We can call such IMUs as a robotic vestibular system. In fact, in humanoid robotics research, the term robotic vestibular system or artificial vestibular system was already in use. However, strictly speaking not in all of the cases inertial sensors may be interpreted as the artificial vestibular system because of the ways the sensors are implemented or used. In [142], one of the first 
attempts to design artificial vestibular system was presented. It was suggested to integrate bi-axial accelerometer and uni-axial gyrometer in a single inertial sensing unit. Later, in [109] a three-axial artificial vestibular system was described. It included a sensing unit with three orthogonal planes. Each plane was equipped with MEMS based accelerometer and gyrometer. Design approaches described in [142] and [109] are similar to the one used in classical sensor engineering when a set of individual inertial sensors were integrated together in a single IMU. An attempt to realise a biomimetic angular rate sensor based on the biomechanical model of the semicircular canals was presented in [32]. However, no further experimental results were presented. A more general point of view to the artificial vestibular system as a part of a multi-sensory system for a robotic rat was presented in [104]. A more thorough example of an artificial vestibular system implementation can be found in [114] where mutual integration of IMU sensing and head control for natural gaze control for was demonstrated for a humanoid robot.

\subsection{Advantages of head stabilisation in human-like robots}

A stabilised robotic head would presumably benefit from the same advantages as those of natural heads, hence we suggest that humanoid robots should also adopt a similar head stabilisation behaviour. Head stabilisation improves sensing robustness of visual and vestibular organs located in the head. It is important for the visual sensory system and gaze stabilisation, as well as it enables the establishment of a stable frame of reference for motion planning within the robots' body.

Among numerous other potential advantages, it is important to mention that a horizontally stabilised head facilitates the estimation of the gravitational vertical during locomotion. Knowledge of the direction of the gravity vector, that is of the gravitational vertical, is essential to achieve stance and locomotion as the gravitational vertical may be poorly estimated from visual cues or based on the kinematic relationship of the robot to the ground. However, when accelerometers cannot distinguish between translational and gravitational components of acceleration when translated and tilted at the same time. We suggest that a stabilised robotic head may improve the quality of inertial measurements of the sensors located in this head. First of all, we suppose that the head stabilised independently from the motion of the trunk will be less affected by external forces and disturbances which may occur during locomotion [51,52]. Second, if the head is stabilised in upright vertical position, then at least one of the axis of inertial sensors will be aligned with gravitational acceleration. This means that a simpler estimation method can be used to process the measurements from inertial sensors, such as accelerometers. In certain situations, it may be assumed that accelerometer located in the upright stabilised head will measure independently gravitational and translational components of acceleration. However, stabilisation of a robotic head with respect to the gravitational verticality requires knowledge of this verticality, and in our case, the only available information related to gravitational verticality can be obtained from inertial sensors, 
in particular from an accelerometer. This means that the control loop has to be closed by the feedback of the measured or estimated vertical orientation of the head. As a result, we obtain an observer-based closed loop control system. The task of the observer is to estimate the vertical orientation of the head based on inertial sensor measurements without the explicit knowledge of translational acceleration of the head. Hence, our head observation-stabilisation system can be considered as self-sufficient or in other words ideothetic.

Idiothetic sensing, or sensing entirely based on states measured with reference to one's own body, is of course not special to robots. In aerospace engineering, flying and rocketing vehicles also use IMU. Long ago, it was noticed that guidance was greatly simplified if the inertial sensors were placed on stabilised platforms [44]. In such systems, the application of fundamental mechanical principles and stabilising control provided engineers with possibilities to establish the gravity referenced Earth's inertial frame without the need for other external references. Ideothetic inertial sensing can be useful to any type of mobile robotic systems [13].

Recently, a concept of top-down control was proposed for humanoid robotics. It was suggested the posture control during locomotion is governed in the head's frame of reference. As the head is stabilised, a stable frame of reference is achieved. Inertial measurements from the vestibular system would be expressed in this frame of reference and would be used for gaze control. Gaze direction anticipates on head orientation which in turn anticipates on the body segments during locomotion $[80,88,15]$ and this behaviour can be used to control steering in assistive walking robots [53]. Following the top-down control organisation, a humanoid robot can be controlled in its stabilised head's frame of reference as it was done in $[130,72]$. In [130], the humanoid robot was teleoperated and its desired walking direction and posture were given to the controller in head's frame. This example of robot control with top-down organization differs from the classical humanoid locomotion control when posture configuration was defined in the world (ground) reference frame. In most of the humanoid walking applications ground is assumed to be flat and rigid, and its inclination has to be known. However, the concept of top-down control organization suggests that the only information which is required is joint (proprioceptive) information of the robot's body with respect to a stabilised head's frame of reference. Head stabilisation also contributes to whole body dynamic stabilisation during locomotion in humanoid robots, as it was demonstrated in [89]. Interestingly, head stabilisation contributed to the stabilisation while the head's mass accounted only for $7 \%$ of the total mass of the body.

\subsection{Current state of research and challenges}

In this final subsection, we would like to re-iterate the importance of head stabilisation for robots through demonstrating some successful implementations. Research on human-like head stabilisation and control has been intensified over last years. In [114] a set of experiments for human-like gaze control for a humanoid robot was presented. Efficiently coordinated head and eye movements 
were achieved through the development of anthropomorphic algorithms based on the head's IMU measurements and position sensing of head-neck and headeye electric motors. Experiments with RoMEO humanoid robot demonstrated that eyes-only, head-only and combined head-eye coordinated movements corresponded to the natural behaviour observed in humans, which in turn contributed to realistic and intuitive human-robot interaction.

The problem of compensating for disturbances induced in a humanoid robot's cameras due to self-generated body movements was approached in [122]. The head's angular velocity measured by an embedded IMU was used to compensate the head's and the eyes' movements, which improved the quality of the robot's optical flow.

An internal model-based adaptive gaze stabilisation for a humanoid robot was proposed in [140]. The internal model was based on the coordination of vestibulocollic and vestibulo-ocular reflexes, and it includes a self-learning and adaptation capabilities. As a result, the experiments with a simulated robotic platform demonstrated that the model was able to stabilise the robot's head independently of the torso movements and at the same time to control gaze for tracking visual targets.

Three head stabilisation control approaches for humanoid robots were described and implemented in [50]. The approaches were two types of inverse kinematic based control and bioinspired adaptive control based on feedback error learning control. All controllers used the robot's head IMU measurements and head-neck joint kinematics information. Additionally to head stabilisation behaviour, vestibulo-ocular and optokinetic reflexes were implemented for the gaze control. Control based on feedback error learning adjusted the mapping of sensory errors into control errors. It showed better performance in head stabilisation behaviour compared to a simple feedback control with inverse kinematics model, demonstrating that nonlinear adaptive techniques are more suitable for anthropomorphic robot control.

Whereas significant progress has been achieved in transferring anthropomorphic behaviours to humanoid robot control, substantial research and development challenges remain. Firstly, resolution of gravito-inertial ambiguity for inertial sensor measurements is problematic for robots with ideothetic sensing and very often employment of external sensors with a fixed frame of reference is required. One more challenging issue, also related to gravito-inertial ambiguity is the existence of several competing gaze-head stabilisation approaches, however, due to technical limitation none of them is mimicking the simplicity of the biological system and achieve sufficient performance. Further development of gaze stabilisation algorithms will also require integration of visual flow based movement compensation, so that accurate visual tracking is achieved while head and gaze are stabilised. Addressing these challenges will enable development of more dynamic, stable and reliable robotic systems, and will be beneficial not only to humanoid robots but also to other types of mobile systems, such as jumping and flying robots. 


\section{Acknowledgment}

I. Farkhatdinov was supported by a fellowship from Ecole Doctorale, Sciences Mécaniques, Acoustique, Electronique et Robotique de Paris (UPMC). Additional funding was provided by the European Research Council, Advanced Grant PATCH, agreement No. 247300 and EU FP7 BALANCE (ICT-601003).

\section{References}

1. A. Ajoudani, J. Lee, A. Rocchi, M. Ferrati, E. M. Hoffman, A. Settimi, D. G. Caldwell, A. Bicchi, and N. G. Tsagarakis. A manipulation framework for compliant humanoid coman: Application to a valve turning task. In 2014 IEEE-RAS International Conference on Humanoid Robots, pages 664-670, Nov 2014.

2. J. H. J. Allum, A. L. Adkin, M. G. Carpenter, M. Held-Ziolkowska, F. Honegger, and K. Pierchala. Trunk sway measures of postural stability during clinical balance tests: effects of a unilateral vestibular deficit. Gait \& Posture, 14(3):227-237, Dec. 2001.

3. J. H. J. Allum, F. Honegger, and C. R. Pfaltz. Afferent Control of Posture and Locomotion, volume 80 of Progress in Brain Research. Elsevier, 1989.

4. W. T. Ang, P. K. Khosla, and C. N. Riviere. Nonlinear regression model of a low-g MEMS accelerometer. IEEE Sensors Journal, 7(1):81-88, Jan. 2007.

5. D. E. Angelaki and K. E. Cullen. Vestibular system: the many facets of a multimodal sense. Annual Reviews of Neuroscience, 31:125-150, 2008.

6. G. Arechavaleta, J.-P. Laumond, H. Hicheur, and A. Berthoz. An optimality principle governing human walking. IEEE T. on Robotics, 24(1):5-14, 2008.

7. T. Asfour, P. Azad, N. Vahrenkamp, K. Regenstein, A. Bierbaum, K. Welke, J. Schröder, and R. Dillmann. Toward humanoid manipulation in human-centred environments. Robotics and Autonomous Systems, 56(1):54-65, Jan. 2008.

8. C. Authie, P. Hilt, S. N'Guyen, A. Berthoz, and D. Bennequin. Differences in gaze anticipation for locomotion with and without vision. Front Hum Neurosci, Jun 9, 2015.

9. A.-J. Baerveldt and R. Klang. A low-cost and low-weight attitude estimation system for an autonomous helicopter. In Proceedings of IEEE International Conference on Intelligent Engineering Systems, pages 391-395. IEEE, 1997.

10. J. Baker, J. Goldberg, and B. Peterson. Spatial and temporal response properties of the vestibulocollic reflex in decerebrate cats. J Neurophysiol, 54(3):735-756, Sept. 1985.

11. G. Baldwin, R. Mahony, and J. Trumpf. A nonlinear observer for 6 dof pose estimation from inertial and bearing measurements. In 2009 IEEE International Conference on Robotics and Automation, pages 2237-2242. IEEE, May 2009.

12. N. Barbour and G. Schmidt. Inertial sensor technology trends. IEEE Sensors Journal, 1(4):332-339, 2001. 
13. B. Barshan and H. Durrant-Whyte. Inertial navigation systems for mobile robots. IEEE T on Robotics and Automation, 11(3):328-342, June 1995.

14. M. Benallegue and F. Lamiraux. Humanoid flexibility deformation can be efficiently estimated using only inertial measurement units and contact information. In 2014 IEEE-RAS International Conference on Humanoid Robots, pages 246251, Nov 2014.

15. D. Bernardin, H. Kadone, D. Bennequin, T. Sugar, M. Zaoui, and A. Berthoz. Gaze anticipation during human locomotion. Exp. Brain Res., 223(1):65-78, Nov. 2012.

16. A. Berthoz. The Brain's Sense of Movement. Harvard University Press, 2000.

17. A. Berthoz, J. Droulez, P. P. Vidal, and K. Yoshida. Neural correlates of horizontal vestibulo-ocular reflex cancellation during rapid eye movements in the cat. $J$. Physiol., 419(1):717-751, Dec. 1989.

18. A. Berthoz, M. G. Jones, and A. Begue. Differential visual adaptation of vertical canal-dependent vestibulo-ocular reflexes. Exp. Brain Res., 44(1), Sept. 1981.

19. A. Berthoz, B. Pavard, and L. Young. Perception of linear horizontal self-motion induced by peripheral vision (linear vection) basic characteristics and visualvestibular interactions. Exp. Brain Res., 23(5), Nov. 1975.

20. A. R. Bisdorff, C. J. Wolsley, D. Anastasopoulos, A. M. Bronstein, and M. A. Gresty. The perception of body verticality (subjective postural vertical) in peripheral and central vestibular disorders. Brain, 119(5):1523-1534, Oct. 1996.

21. J. Borah, L. R. Young, and R. E. Curry. Optimal estimator model for human spatial orientation. Annals of the New York Academy of Sciences, 545(1 Representatio):51-73, Dec. 1988.

22. S. Bras, R. Cunha, J. F. Vasconcelos, C. Silvestre, and P. Oliveira. A nonlinear attitude observer based on active vision and inertial measurements. IEEE T. on Robotics, 27(4):664-677, 2011.

23. A. M. Bronstein. Evidence for a vestibular input contributing to dynamic head stabilization in man. Acta oto-laryngologica, 105(1-2):1-6, July 1998.

24. R. A. Brooks and C. Breazeal. The cog project: building a humanoid robot. In Computation for Metaphors, Analogy, and Agents. Lecture Notes in Computer Science, volume 1562, pages 52-87. 1999.

25. J. J. Buchanan and F. B. Horak. Vestibular loss disrupts control of head and trunk on a sinusoidally moving platform. Journal of vestibular research : equilibrium $\&$ orientation, 11(6):371-89, 2002.

26. E. Bums, R. Homing, W. Herb, J. Zook, and H. Guckel. Resonant microibeam accelerometers. In Proceedings of the International Solid-State Sensors and Actuators Conference - TRANSDUCERS '95, volume 2, pages 659-662. IEEE, 1995.

27. T. Buschmann, S. Lohmeier, and H. Ulbrich. Humanoid robot lola: design and walking control. Journal of physiology-Paris, 103(3-5):141-148, 2009.

28. J. P. Carey and C. C. D. Santina. Principles of applied vestibular physiology. In Cummings Otolaryngology - Head and Neck Surgery, chapter 163. Mosby, 2005. 
29. Y.-H. Chang, Y. Oh, D. Kim, and S. Hong. Balance control in whole body coordination framework for biped humanoid robot mahru-r. In RO-MAN 2008 - The 17th IEEE International Symposium on Robot and Human Interactive Communication, pages 401-406. IEEE, Aug. 2008.

30. W. G. Chase and H. H. Clark. Semantics in the perception of verticality. British Journal of Psychology, 62(3):311-326, Aug. 1971.

31. G. Cheng, S.-H. Hyon, J. Morimoto, A. Ude, J. G. Hale, G. Colvin, W. Scroggin, and S. C. Jacobsen. Cb: a humanoid research platform for exploring neuroscience. Advanced Robotics, 21(10):1097-1114, Jan. 2007.

32. G. Ciaravella, C. Laschi, and P. Dario. Biomechanical modeling of semicircular canals for fabricating a biomimetic vestibular system. In IEEE Engineering in Medicine and Biology Society, volume 1, pages 1758-1761, Jan. 2006.

33. J. L. Crassidis, F. L. Markley, and Y. Cheng. Survey of nonlinear attitude estimation methods. Journal of Guidance, Control, and Dynamics, 30(1):12-28, Jan. 2007.

34. T. W. Cronin, M. R. Kinloch, and G. H. Olsen. Head-bobbing behavior in walking whooping cranes (grus americana) and sandhill cranes (grus canadensis). Journal of Ornithology, 148(S2):563-569, Aug. 2007.

35. K. E. Cullen. The vestibular system: multimodal integration and encoding of self-motion for motor control. Trends in neurosciences, 35(3):185-96, Mar. 2012.

36. I. S. Curthoys, C. H. Markham, and E. J. Curthoys. Semicircular duct and ampulla dimensions in cat, guinea pig and man. Journal of morphology, 151(1):1734, Jan. 1977.

37. R. David, A. Stoessel, A. Berthoz, F. Spoor, and D. Bennequin. Assessing morphology and function of the semicircular duct system: introducing new in-situ visualization and software toolbox. Scientific reports, 6, 2016.

38. M. N. Davies and P. R. Green. Head-bobbing during walking, running and flying: relative motion perception in the pigeon. J. Exp. Biol., 138(1):71-91, Sept. 1988.

39. H. De Vries. The mechanics of the labyrinth otoliths. Acta oto-laryngologica, 38(3):262-73, June 1951.

40. M. Dieterich and T. Brandt. Vestibulo-ocular reflex. Current opinion in neurology, 8(1):83-8, Feb. 1995.

41. M. Dimiccoli, B. Girard, A. Berthoz, and D. Bennequin. A functional explanation of otolith geometry. J Comput Neurosci, 35(2), 2013.

42. D. C. Dunbar. Stabilization and mobility of the head and trunk in wild monkeys during terrestrial and flat-surface walks and gallops. J. Exp. Biol., 207(6):10271042, Feb. 2004.

43. D. C. Dunbar, J. M. Macpherson, R. W. Simmons, and A. Zarcades. Stabilization and mobility of the head, neck and trunk in horses during overground locomotion: comparisons with humans and other primates. J. Exp. Biol., 211(Pt 24):3889-907, Dec. 2008. 
44. R. C. Duncan and A. L. F. S. J. Gunnersen. Inertial guidance, navigation, and control systems. Journal of Spacecraft and Rockets, 1(6):577-587, 1964.

45. M. O. Ernst and H. H. Bülthoff. Merging the senses into a robust percept. Trends in cognitive sciences, 8(4):162-9, Apr. 2004.

46. M. Euston, P. Coote, R. Mahony, and T. Hamel. A complementary filter for attitude estimation of a fixed-wing uav. In 2008 IEEE/RSJ International Conference on Intelligent Robots and Systems, pages 340-345. IEEE, Sept. 2008.

47. K. Ezure, S. Sasaki, Y. Uchino, and V. J. Wilson. Frequency-response analysis of vestibular-induced neck reflex in cat. ii. functional significance of cervical afferents and polysynaptic descending pathways. J Neurophysiol, 41(2):459-471, Mar. 1978.

48. M. F. Fallón, M. Antone, N. Roy, and S. Teller. Drift-free humanoid state estimation fusing kinematic, inertial and lidar sensing. In 2014 IEEE-RAS International Conference on Humanoid Robots, pages 112-119, Nov 2014.

49. E. Falotico, N. Cauli, K. Hashimoto, P. Kryczka, A. Takanishi, P. Dario, A. Berthoz, and C. Laschi. Head stabilization based on a feedback error learning in a humanoid robot. In 2012 IEEE RO-MAN: The 21st IEEE International Symposium on Robot and Human Interactive Communication, pages 449-454. IEEE, Sept. 2012.

50. E. Falotico, N. Cauli, P. Kryczka, K. Hashimoto, A. Berthoz, A. Takanishi, P. Dario, and C. Laschi. Head stabilization in a humanoid robot: models and implementations. Auton. Robots, 41(2):349-365, 2017.

51. I. Farkhatdinov, V. Hayward, and A. Berthoz. On the benefits of head stabilization with a view to control balance and locomotion in humanoids. In 11th IEEE-RAS International Conference on Humanoid Robots 2011, pages 147-152, Bled, Slovenia, 2011. IEEE.

52. I. Farkhatdinov, H. Michalska, A. Berthoz, and V. Hayward. Modeling verticality estimation during locomotion. In Romansy 19, Robot Design, Dynamics and Control, volume 544 of CISM International Centre for Mechanical Sciences, pages 359-366, Paris, France, 2012. Springer.

53. I. Farkhatdinov, N. Roehri, and E. Burdet. Anticipatory detection of turning in humans for intuitive control of robotic mobility assistance. Bioinspiration $\mathcal{E}$ Biomimetics, 12(5), 2017.

54. H. Fourati, N. Manamanni, L. Afilal, and Y. Handrich. A nonlinear filtering approach for the attitude and dynamic body acceleration estimation based on inertial and magnetic sensors: bio-logging application. IEEE Sensors Journal, 11(1):233-244, Jan. 2011.

55. B. J. Frost. The optokinetic basis of head-bobbing in the pigeon. J. Exp. Biol., 74:187-195, 1978.

56. M. Fujita. Head bobbing and the body movement of little egrets (egretta garzetta) during walking. Journal of comparative physiology. A, Neuroethology, sensory, neural, and behavioral physiology, 189(1):53-8, Jan. 2003.

57. M. J. G, A. Berthoz, and B. Segal. Adaptive modification of the vestibulo-ocular reflex by mental effort in darkness. Exp. Brain Res., 56(1), Aug. 1984. 
58. S. Gay, A. Ijspeert, and J. Santos Victor. Predictive gaze stabilization during periodic locomotion based on adaptive frequency oscillators. In 2012 IEEE International Conference on Robotics and Automation, pages 271-278. IEEE, May 2012.

59. D. Gebre-Egziabher, R. Hayward, and J. Powell. A low-cost gps/inertial attitude heading reference system (ahrs) for general aviation applications. In IEEE 1998 Position Location and Navigation Symposium (Cat. No.98CH36153), pages 518525. IEEE, 1998.

60. J. Geen and D. Krakauer. Rate-sensing gyroscope. Technical report, ADI Micromachined Products Division, 2003.

61. J. Goldberg and B. W. Peterson. Reflex and mechanical contributions to head stabilization in alert cats. J Neurophysiol, 56(3):857-875, Sept. 1986.

62. D. Gouaillier, V. Hugel, P. Blazevic, C. Kilner, J. Monceaux, P. Lafourcade, B. Marnier, J. Serre, and B. Maisonnier. Mechatronic design of nao humanoid. In 2009 IEEE International Conference on Robotics and Automation, pages 769-774. IEEE, May 2009.

63. M. Grassi. Attitude determination and control for a small remote sensing satellite. Acta Astronautica, 40(9):675-681, May 1997.

64. R. Grasso, P. Prévost, Y. P. Ivanenko, and A. Berthoz. Eye-head coordination for the steering of locomotion in humans: an anticipatory synergy. Neuroscience Letters, 253(2):115-118, Sept. 1998.

65. P. R. Green. Head orientation and trajectory of locomotion during jumping and walking in domestic chicks. Brain, behavior and evolution, 51(1):48-58, Jan. 1998.

66. P. Greiff, B. Antkowiak, J. Campbell, and A. Petrovich. Vibrating wheel micromechanical gyro. In Proc. of Position, Location and Navigation Symposium PLANS '96, pages 31-37. IEEE, 1996.

67. M. Grewal, V. Henderson, and R. Miyasako. Application of kalman filtering to the calibration and alignment of inertial navigation systems. IEEE T on Automatic Control, 36(1):3-13, 1991.

68. J. J. Groen. Cupulometry. The Laryngoscope, 67(9):894-905, Sept. 1957.

69. D. Guitton, R. Kearney, N. Wereley, and B. Peterson. Visual, vestibular and voluntary contributions to human head stabilization. Exp. Brain Res., 64(1), Sept. 1986.

70. T. Hain, T. Ramaswamy, and M. Hilmann. Anatomy and physiology of vestibular system. In Herdman SJ. Vestibular Rehabilitation, chapter 1. 2007.

71. J. Hale and G. Cheng. Full-body compliant human -humanoid interaction: Balancing in the presence of unknown external forces. IEEE T on Robotics, 23(5):884898, Oct. 2007.

72. K. Hashimoto, H. Kang, M. Nakamura, E. Falotico, H. Lim, A. Takanishi, C. Laschi, P. Dario, and A. Berthoz. Realization of biped walking on soft ground with stabilization control based on gait analysis. In Proceedings of the 2012 IEEE/RSJ International Conference on Intelligent Robots and Systems, Oct 2014. 
73. H. Hicheur, S. Vieilledent, and A. Berthoz. Head motion in humans alternating between straight and curved walking path: combination of stabilizing and anticipatory orienting mechanisms. Neuroscience letters, 383(1-2):87-92, 2005.

74. M. Hirose and K. Ogawa. Honda humanoid robots development. Philosophical transactions. Series A, Mathematical, physical, and engineering sciences, 365(1850):11-9, Jan. 2007.

75. J. Hulk and L. B. W. Jongkees. The turning test with small regulable stimuli. The Journal of Laryngology $\&$ Otology, 62(02):70-75, 1948.

76. M. Igarashi, T. O-Uchi, and B. R. Alford. Volumetric and dimensional measurements of vestibular structures in the squirrel monkey. Acta oto-laryngologica, 91(5-6):437-44, 1981.

77. I. Israel, R. Grasso, P. Georges-Francois, T. Tsuzuku, and A. Berthoz. Spatial memory and path integration studied by self-driven passive linear displacement. i. basic properties. J Neurophysiol, 77(6):3180-3192, June 1997.

78. I. Israël, S. Rivaud, B. Gaymard, A. Berthoz, and C. Pierrot-Deseilligny. Cortical control of vestibular-guided saccades in man. Brain, 118(5):1169-1183, Oct. 1995.

79. Y. Jun, R. Ellenburg, and P. Oh. From concept to realization: designing miniature humanoids for running. J. on Systemics, Cybernetics and Informatics, 2010.

80. H. Kadone, D. Bernardin, D. Bennequin, and A. Berthoz. Gaze anticipation during human locomotion - top-down organization that may invert the concept of locomotion in humanoid robots. 19th International Symposium in Robot and Human Interactive Communication, pages 552-557, Sept. 2010.

81. S. Kagami, M. Mochimaru, Y. Ehara, N. Miyata, K. Nishiwaki, T. Kanade, and H. Inoue. Measurement and comparison of humanoid h7 walking with human being. Robotics and Autonomous Systems, 48(4):177-187, Oct. 2004.

82. R. E. Kalman. A new approach to linear filtering and prediction problems 1. Transactions of the ASME Journal of Basic Engineering, 82(Series D):35-45, 1960.

83. G. Katzir, E. Schechtman, N. Carmi, and D. Weihs. Head stabilization in herons. Journal of Comparative Physiology A: Sensory, Neural, and Behavioral Physiology, 187(6):423-432, July 2001.

84. E. A. Keshner, T. C. Hain, and K. J. Chen. Predicting control mechanisms for human head stabilization by altering the passive mechanics. Journal of vestibular research : equilibrium \& orientation, 9(6):423-34, Jan. 1999.

85. J.-Y. Kim, I.-W. Park, and J.-H. Oh. Experimental realization of dynamic walking of the biped humanoid robot khr-2 using zero moment point feedback and inertial measurement. Advanced Robotics, 20(6):707-736, Jan. 2006.

86. P. Kryczka, E. Falotico, K. Hashimoto, H. Lim, A. Takanishi, C. Laschi, P. Dario, and A. Berthoz. Implementation of a human model for head stabilization on a humanoid platform. In 2012 4th IEEE RAS $\&$ EMBS International Conference on Biomedical Robotics and Biomechatronics (BioRob), pages 675-680. IEEE, June 2012. 
87. D. Lapadatu, S. Habibi, B. Reppen, G. Salomonsen, and T. Kvisteroy. Dualaxes capacitive inclinometer/low-g accelerometer for automotive applications. In 14th IEEE International Conference on Micro Electro Mechanical Systems, pages 34-37. IEEE, 2001.

88. J.-P. Laumond, G. Arechavaleta, T.-V.-A. Truong, H. Hicheur, Q.-C. Pham, and A. Berthoz. The words of the human locomotion. In M. Kaneko and Y. Nakamura, editors, Robotics Research, volume 66 of Springer Tracts in Advanced Robotics, pages 35-47. Springer, 2011.

89. J.-P. Laumond, M. Benallegue, J. Carpentier, and A. Berthoz. The yoyo-man. The International Journal of Robotics Research, page 0278364917693292, 2017.

90. J. Laurens and J. Droulez. Bayesian processing of vestibular information. Biological cybernetics, 96(4):389-404, Apr. 2007.

91. J. Leavitt, A. Sideris, and J. Bobrow. High bandwidth tilt measurement using low-cost sensors. IEEE/ASME T on Mechatronics, 11(3):320-327, June 2006.

92. C.-H. Lin and S.-M. Kuo. High-performance inclinometer with wide-angle measurement capability without damping effect. In 2007 IEEE 20th International Conference on Micro Electro Mechanical Systems (MEMS), pages 585-588. IEEE, 2007.

93. S. Lohmeier, T. Buschmann, and H. Ulbrich. System design and control of anthropomorphic walking robot lola. IEEE/ASME T on Mechatronics, 14(6):658-666, Dec. 2009 .

94. M. Lopes, A. Bernardino, J. Santos-Victor, K. Rosander, and C. von Hofsten. Biomimetic eye-neck coordination. In 2009 IEEE 8th International Conference on Development and Learning. IEEE, 2009.

95. D. G. Luenberger. Observing the state of a linear system. IEEE T on Military Electronics, 8(2):74-80, 1964.

96. P. R. MacNeilage, N. Ganesan, and D. E. Angelaki. Computational approaches to spatial orientation: from transfer functions to dynamic bayesian inference. Journal of neurophysiology, 100(6):2981-96, Dec. 2008.

97. A. B. A. Manaf, K. Nakamura, J. Onishi, and Y. Matsumoto. One-side-electrodetype fluid-based inclinometer combined with cmos circuitry. In 2007 IEEE Sensors, pages 844-847. IEEE, 2007.

98. D. Manchester, M. Woollacott, N. Zederbauer-Hylton, and O. Marin. Visual, vestibular and somatosensory contributions to balance control in the older adult. Journal of Gerontology, 44(4):M118-M127, July 1989.

99. D. M. Merfeld, L. Zupan, and R. J. Peterka. Humans use internal models to estimate gravity and linear acceleration. Nature, 398(6728):615-8, Apr. 1999.

100. D. M. Merfeld and L. H. Zupan. Neural processing of gravitoinertial cues in humans. iii. modeling tilt and translation responses. J Neurophysiol, 87(2):819833, Feb. 2002.

101. D. M. Merfeld, L. H. Zupan, and C. A. Gifford. Neural processing of gravitoinertial cues in humans. ii. influence of the semicircular canals during eccentric 
rotation. J Neurophysiol, 85(4):1648-1660, Apr. 2001.

102. U. Mescheder and S. Majer. Micromechanical inclinometer. Sensors and Actuators A: Physical, 60(1-3):134-138, May 1997.

103. N. Metni, J.-M. Pflimlin, T. Hamel, and P. Souères. Attitude and gyro bias estimation for a vtol uav. Control Engineering Practice, 14(12):1511-1520, Dec. 2006.

104. J.-A. Meyer, A. Guillot, B. Girard, M. Khamassi, P. Pirim, and A. Berthoz. The psikharpax project: towards building an artificial rat. Robotics and Autonomous Systems, 50(4):211-223, Mar. 2005.

105. P. Mottier and P. Pouteau. Solid state optical gyrometer integrated on silicon. Electronics Letters, 33(23), Nov. 1997.

106. R. Necker. Head-bobbing of walking birds. Journal of comparative physiology. A, Neuroethology, sensory, neural, and behavioral physiology, 193(12):1177-83, Dec. 2007.

107. J. Niven and W. Hixson. Frequency response of the human semicircular canals: I. steady-state ocular nystagmus response to high-level sinusoidal angular rotations. Technical report, NASA Naval School of Aviation Medicine., 1961.

108. M. J. Novack. Design and fabrication of a thin film micromachined accelerometer. PhD thesis, Massachusetts Institute of Technology, 1992.

109. F. Paian, C. Laschi, H. Miwa, E. Guglielmelli, P. Dario, and A. Takanishi. Design and development of a biologically-inspired artificial vestibular system for robot heads. In 2004 IEEE/RSJ International Conference on Intelligent Robots and Systems (IROS), volume 2, pages 1317-1322. IEEE, 2004.

110. F. Panerai, G. Metta, and G. Sandini. Learning visual stabilization reflexes in robots with moving eyes. Neurocomputing, 48(1-4):323-337, Oct. 2002.

111. A. Paramiggiani, M. Maggiali, L. Natale, F. Nori, A. Schmitz, N. Tsagarakis, J. S. Victor, F. Becchi, G. Sandini, and G. Metta. The design of the icub humanoid robot. International Journal of Humanoid Robotics, page 1250027, Nov. 2012.

112. I.-W. Park, J.-Y. Kim, J. Lee, and J.-H. Oh. Mechanical design of humanoid robot platform khr-3 (kaist humanoid robot - 3: Hubo). In 5th IEEE-RAS International Conference on Humanoid Robots, 2005., pages 321-326. IEEE, 2005.

113. P. Pascal, Y. Ivanenko, R. Grasso, and A. Berthoz. Spatial invariance in anticipatory orienting behaviour during human navigation. Neuroscience Letters, 339(3):243-247, Mar. 2003.

114. N. Pateromichelakis, A. Mazel, M. Hache, T. Koumpogiannis, R. Gelin, B. Maisonnier, and A. Berthoz. Head-eyes system and gaze analysis of the humanoid robot romeo. In Intelligent Robots and Systems (IROS 2014), 2014 IEEE/RSJ International Conference on, pages 1374-1379. IEEE, 2014.

115. T. Pozzo, A. Berthoz, and L. Lefort. Head stabilisation during various locomotor tasks in humans. Exp. Brain Res., 82(1):97-106, 1990.

116. T. Pozzo, A. Berthoz, L. Lefort, and E. Vitte. Head stabilization during various locomotory tasks in humans ii. patients with bilateral vestibular deficits. Exp. 
Brain Res., 85:208-217, 1991.

117. T. Pozzo, Y. Levik, and A. Berthoz. Head and trunk movements in the frontal plane during complex dynamic equilibrium tasks in humans. Exp. Brain Res., 106(2):327-338, 1995.

118. R. Puers and S. Reyntjens. Design and processing experiments of a new miniaturized capacitive triaxial accelerometer. Sensors and Actuators A: Physical, 68(1-3):324-328, June 1998.

119. J. Qian, B. Fang, W. Yang, X. Luan, and H. Nan. Accurate tilt sensing with linear model. IEEE Sensors Journal, 11(10):2301-2309, Oct. 2011.

120. H. Rehbinder and X. Hu. Drift-free attitude estimation for accelerated rigid bodies. Automatica, 40(4):653-659, Apr. 2004.

121. H. Reisine, J. I. Simpson, and V. Henn. A geometric analysis of semicircular canals and induced activity in their peripheral afferents in the rhesus monkey. Annals of the New York Academy of Sciences, 545:10-20, Jan. 1988.

122. A. Roncone, U. Pattacini, G. Metta, and L. Natale. Gaze stabilization for humanoid robots: A comprehensive framework. In Humanoid Robots (Humanoids), 2014 14th IEEE-RAS International Conference on, pages 259-264. IEEE, 2014.

123. N. Rotella, M. Bloesch, L. Righetti, and S. Schaal. State estimation for a humanoid robot. In 2014 IEEE/RSJ International Conference on Intelligent Robots and Systems, pages 952-958, Sept 2014.

124. Y. Sakagami, R. Watanabe, C. Aoyama, S. Matsunaga, N. Higaki, and K. Fujimura. The intelligent asimo: system overview and integration. In IEEE/RSJ International Conference on Intelligent Robots and System, volume 3, pages 24782483. IEEE, 2002.

125. M. Schwarz, T. Rodehutskors, M. Schreiber, and S. Behnke. Hybrid drivingstepping locomotion with the wheeled-legged robot momaro. In 2016 IEEE International Conference on Robotics and Automation (ICRA), pages 5589-5595, May 2016.

126. P. Selva and C. M. Oman. Relationships between observer and kalman filter models for human dynamic spatial orientation. Journal of vestibular research : equilibrium \& orientation, 22(2):69-80, Jan. 2012.

127. N. T. Shepard, S. A. Telian, M. Smith-Wheelock, and A. Raj. Vestibular and balance rehabilitation therapy. The Annals of otology, rhinology, and laryngology, 102(3 Pt 1):198-205, Mar. 1993.

128. T. Shibata and S. Schaal. Biomimetic gaze stabilization based on feedback-errorlearning with nonparametric regression networks. Neural Networks, 14(2):201216, Mar. 2001.

129. T. Shibata and S. Vijayakumar. Humanoid oculomotor control based on concepts of computational neuroscience. In IEEE-RAS Int. Conf. on Humanoid robots, Japan, 2001.

130. M. N. Sreenivasa, P. Soueres, J.-P. Laumond, and A. Berthoz. Steering a humanoid robot by its head. In 2009 IEEE/RSJ International Conference on Intel- 
ligent Robots and Systems, pages 4451-4456. IEEE, Oct. 2009.

131. B. J. Stephens and C. G. Atkeson. Dynamic balance force control for compliant humanoid robots. In 2010 IEEE/RSJ International Conference on Intelligent Robots and Systems, pages 1248-1255. IEEE, Oct. 2010.

132. N. Sugimoto, J. Morimoto, S.-H. Hyon, and M. Kawato. The emosaic model for humanoid robot control. Neural networks : the official journal of the International Neural Network Society, 29-30(null):8-19, May 2012.

133. M. S. Svendsen, J. Helbo, M. R. Hansen, D. B. Popovic, J. Stoustrup, and M. M. Pedersen. Aau-bot1: a platform for studying dynamic, life-like walking. Applied Bionics and Biomechanics, 6(3-4):285-299, Dec. 2009.

134. K. A. Tahboub. Biologically-inspired postural and reaching control of a multisegment humanoid robot. International Journal of Biomechatronics and Biomedical Robotics, 1(3):175-190, 2011.

135. S. Trimpe and R. D'Andrea. Accelerometer-based tilt estimation of a rigid body with only rotational degrees of freedom. In 2010 IEEE International Conference on Robotics and Automation, pages 2630-2636. IEEE, May 2010.

136. N. Troje and B. Frost. Head-bobbing in pigeons: how stable is the hold phase? J. Exp. Biol., 203(5):935-940, Mar. 2000.

137. N. G. Tsagarakis, G. Metta, G. Sandini, D. Vernon, R. Beira, F. Becchi, L. Righetti, J. Santos-Victor, A. J. Ijspeert, M. C. Carrozza, and D. G. Caldwell. icub: the design and realization of an open humanoid platform for cognitive and neuroscience research. Advanced Robotics, 21(10):1151-1175, Jan. 2007.

138. J. Vaganay, M. Aldon, and A. Fournier. Mobile robot attitude estimation by fusion of inertial data. In Proceedings IEEE International Conference on Robotics and Automation, pages 277-282. IEEE Comput. Soc. Press, 1993.

139. W. C. Van Buskirk, R. G. Watts, and Y. K. Liu. The fluid mechanics of the semicircular canals. Journal of Fluid Mechanics, 78(01):87-98, 1976.

140. L. Vannucci, S. Tolu, E. Falotico, P. Dario, H. H. Lund, and C. Laschi. Adaptive gaze stabilization through cerebellar internal models in a humanoid robot. In Biomedical Robotics and Biomechatronics (BioRob), 2016 6th IEEE International Conference on, pages 25-30. IEEE, 2016.

141. J. Vasconcelos, R. Cunha, C. Silvestre, and P. Oliveira. A nonlinear position and attitude observer on se(3) using landmark measurements. Systems \& Control Letters, 59(3-4):155-166, Mar. 2010.

142. P. H. Veltink, H. J. Luinge, B. J. Kooi, C. T. M. Baten, and P. Slycke. The artificial vestibular system - design of a tri-axial inertial sensor system and its application in the study of human movement. In Proceedings of the International Society for Postural and Gait Research, number 1, 2001.

143. M. Vukobratovic and D. Juricic. Contribution to the synthesis of biped gait. IEEE T on Biomedical Engineering, BME-16(1):1-6, Jan. 1969.

144. G. Welch and E. Foxlin. Motion tracking: no silver bullet, but a respectable arsenal. IEEE Computer Graphics and Applications, 22(6):24-38, Nov. 2002. 
145. D. Winter. Human balance and posture control during standing and walking. Gait \& Posture, 3(4):193-214, Dec. 1995.

146. D. A. Winter, A. E. Patla, F. Prince, M. Ishac, and K. Gielo-Perczak. Stiffness control of balance in quiet standing. J Neurophysiol, 80(3):1211-1221, Sept. 1998.

147. H. Wongsuwarn and D. Laowattana. Experimental study for a fibo humanoid robot. In IEEE Conference on Robotics, Automation and Mechatronics, 2006.

148. Y. Xiang, S. B. Yakushin, M. Kunin, T. Raphan, and B. Cohen. Head stabilization by vestibulocollic reflexes during quadrupedal locomotion in monkey. Journal of neurophysiology, 100(2):763-80, Aug. 2008.

149. A. P. Yelnik. Perception of verticality after recent cerebral hemisphericstroke. Stroke, 33(9):2247-2253, Sept. 2002.

150. R. Yotter, R. Baxter, S. Ohno, S. Hawley, and D. Wilson. On a micromachined fluidic inclinometer. In TRANSDUCERS '03. 12th Int. Conf. on Solid-State Sensors, Actuators and Microsystems, volume 2, pages 1279-1282. IEEE, 2003.

151. L. H. Zupan, R. J. Peterka, and D. M. Merfeld. Neural processing of gravitoinertial cues in humans. i. influence of the semicircular canals following postrotatory tilt. J Neurophysiol, 84(4):2001-2015, Oct. 2000. 